\title{
Assessing perioperative risk and fitness in patients undergoing elective major non-cardiac surgery
}

\author{
C.W. Lai, MBBS, MRCS ${ }^{1}$, K.B. Hosie, MBChB FRCSEd MD ${ }^{2}$ \\ 1 Clinical Research Fellow, Derriford Hospital, Plymouth, UK \\ 2 Consultant Surgeon, Derriford Hospital, Plymouth and Head of Peninsula School of Surgery, Peninsula Deanery, \\ Plymouth, UK
}

Key words: Risk Assessment; Elective Surgery;

Morbidity; Mortality.

\section{Introduction}

Perioperative care of patients undergoing major surgery is an area of medicine that has always received much interest, but to date has yet to reach a consensus on "the best practice". It remains a challenge for the clinical team to provide the best perioperative care in order to minimise postoperative morbidity and mortality.

The volume of major surgery carried out annually worldwide is very large. The implication for public health and cost are significant and require serious attention. It has been estimated that 234.2 million major surgical procedures are undertaken every year worldwide [1]. It is further estimated that major morbidity rate in the developed countries is between $3-16 \%$, and that of death or permanent disability is about $0.4-0.8 \%$ [1]. In the UK, 2.3 million surgical procedures are performed each year, with a mortality of $1.4 \%$ [2]. Mortality rates amongst the high-risk surgical population is between $5.8-35 \%$ [2].

\section{Why do we need to risk stratify patients pre- operatively?}

Major surgery alters the patient's normal physiology and generates a strong systemic inflammatory response. This requires increased cardiac output and oxygen delivery in order to meet the increased oxygen demand. Patients who do not have the physiological

Correspondence: Ken Hosie, Consultant Surgeon, Derriford Hospital, Plymouth, UK

Email: kenhosie@gmail.com

The Sri Lanka Journal of Surgery 2012; 30(1):1-4. reserve to meet the metabolic demand of major surgery are at an increased risk of post-operative morbidity and mortality [3, 4].

Identifying high-risk surgical patients pre-operatively is important to allow optimization and careful perioperative planning in order to achieve the best outcome post-operatively. In the process of identifying the high-risk surgical patient, surgical factors such as duration and complexity of surgery are just as important as patient factors such as cardiorespiratory fitness and co-existing morbidity.

\section{What are the available methods in stratifying high- risk surgical patient?}

There are many methods described and used to attempt to identify the high-risk surgical patient preoperatively but to date, there is no consensus as to the what is "gold-standard" [3]. Evidence suggests that despite the large population of high-risk patients undergoing major surgery, clinicians are not identifying these patients and hence may fail to provide optimum peri-operative care [2].

\section{Pre-operative assessment to identify known risk factors}

This is the simplest method and is usually carried out in a clinical setting to identify risks factors such as age over 65 years, existing comorbid disease, and extent of surgery. This may identify the very fit patients but is very non-specific and would fail to clearly define or predict post-operative co-morbidities. 


\section{Duke Activity Status Index (DASI) questionnaire}

The use of the American College of Cardiology and American Heart Association (ACC/AHA) guidelines on perioperative cardiovascular evaluation use a derivative of the DASI questionnaire to estimate functional status [5]. This is an algorithm of questions which quantifies patients' functional status according to their existing exercise ability or tolerance by assigning metabolic equivalent tasks (METS) to their level of daily activities. However, as this method was developed with cardiology patients, its use has not been validated in a general surgical population [6].

\section{Functional walk tests}

There are various types of walk tests described as a tool for evaluating functional status and capacity [7]. These tests are an objective measurement of exercise tolerance and give an estimate of oxygen consumption. They require less specialist equipment and expertise, and hence are simpler and cheaper to use.

In a timed-based test walk test (eg. 2 minute walk test [2MWT], 6 minute walk test [6MWT] and 12 minute walk test $[12 \mathrm{MWT}]$ ), patients are instructed to walk for the duration of the allocated time and the distance walked is recorded [8].

A self-paced walk test (SPWT) requires a patient to walk a determined distance at various speeds. An average speed is calculated for each walking pace. This is a velocity-determined test [9].

During incremental shuttle walk test (ISWT), a patient walks at increasing speeds after every minute and the test is terminated when the patient reaches exhaustion [10]. This measures the maximal capacity of the patient. The endurance shuttle walk test complements the ISWT and measures the patient's ability to use their exercise capacity [11].

Whilst most of these tests have been used to determine capacity and monitoring treatment response in patients with chronic obstructive airway disease and cardiac disease [12,13], its use in the general surgical population is somewhat limited. This is mainly due to the significant proportion of elderly patients, who have limitations in their ability to walk.

\section{Echocardiography}

Trans-thoracic echocardiography is a simple noninvasive test, which assess the systolic and diastolic wall motion. However, there is poor correlation between trans-thoracic echocardiography findings and functional capacity. Therefore, the use of transthoracic echocardiography in the assessment of cardiac risk prior to non-cardiac surgery is not recommended [14].

Radionuclide ventriculography is also used to assess ejection fraction. This has also been shown to correlate poorly with exercise capacity and peak oxygen uptake $[15,16]$.

Dobutamine stress echocardiography has a high sensitivity and specificity for detection of myocardial ischaemia but does not give an objective measurement of functional capacity and is not appropriate for preoperative screening.

\section{Plasma biomarkers}

Preoperative blood sampling to measure plasma biomarkers is still a new and emerging concept. Markers used include B-type natriuretic peptide, glomerular filtration rate and cardiac troponins. These markers reflect levels of pre-existing organ dysfunction that predispose to postoperative complications [17].

\section{Cardiopulmonary exercise test (CPET)}

Cardiopulmonary exercise test (CPET) provides a comprehensive assessment of cardiorespiratory fitness. It examines the ability of the patients' physiology and cardiorespiratory systems to meet the oxygen demands during tissue stress in major surgery.

CPET requires a patient to cycle on a static ergometric bicycle at increasing workloads. This reduces the stress on joints and is much more compliant to patients than other forms of exercise testing such as walking. Respiratory gas analysis and cardiac function by means of electrocardiography is recorded. The key measurements include peak oxygen consumption (V02 peak) and anaerobic threshold (AT). The AT is easy to measure and is determined with high accuracy. This is the point when anaerobic metabolism takes 
over aerobic metabolism, reflecting the inadequacy of oxygen delivery to meet energy demand. Low AT has been shown to correlate poorly with postoperative outcomes [18, 19]. An AT $>11 \mathrm{mlO} 2 / \mathrm{min} / \mathrm{kg}$ has been demonstrated to predict postoperative survival with high sensitivity and specificity $[18,19]$.

Although CPET is an easily repeatable test which determines functional capacity with high accuracy, it requires dedicated equipment and staffing.

\section{How will risk stratification change perioperative care?}

Preoperative fitness level and risk stratification provides invaluable information. It allows strategic perioperative management of a patient. This is part of the clinical governance and helps constantly improve the quality framework for excellent perioperative patient care and postoperative outcome. Various care pathways are identified in this process, which involve multidisciplinary team working.

This allows preoperative optimisation and conditioning in high-risk patients requiring major surgery. This may involve medical or drug treatment, respiratory therapy and interventional cardiology treatment. A multimodal approach to perioperative care, the enhanced recovery program (ERAS) is now widely used. It is associated with rapid postoperative recovery and reduced length of hospital stay [20]. Postoperative level of care can be better planned. This may involve elective admission to intensive care units or high dependency units. A study of the high-risk surgical population in the United Kingdom has shown that we underestimate the size of this population [2]. It has also shown that mortality rate was highest in the group of patients admitted to the intensive care unit following initial care on a standard ward postoperatively. Although elective postoperative care in an intensive care setting seems to be beneficial, evidence that this would improve life expectancy is still lacking.

\section{Summary}

Identification of high risk patients resulting in effective perioperative optimisation and planned perioperative support is likely to reduce morbidity and mortality. The use of appropriate and sometimes multimodal assessment within a multidisciplinary team is likely to provide the most information which will allow the best outcomes.

The best strategies and clinical benefits are still not defined.

\section{References}

1. Weiser TG, Regenbogen SE, Thompson KD, Haynes AB, Lipsitz SR, Berry WR, et al. An estimation of the global volume of surgery: a modeling strategy based on available data. Lancet 2008; 63:695-700.

2. Pearse RM, Harrison DA, James P, Watson D, Hinds C, Rhodes A, Grounds M, Bennett ED. Identification and characterization of the highrisk surgical population in the United Kingdom. Critical Care 2006; 10:R81.

3. Wilson JT, Davies SJ. Pre-operative optimization of the high-risk surgical patient. Br J Anaesthesia 2004; 93(1): 121-8.

4. Shoemaker WC, Appel PL, Kram HB. Role of oxygen debt in the development of organ failure, sepsis and death in high risk surgical patients. Chest 1992; 102: 208-15.

5. Fleisher LA, Beckman JA, Brown KA, et al. ACC/AHA 2007 guidelines on perioperative cardiovascular evaluation and care for noncardiac surgery: a report of the American College of Cardiology/American Heart Association Task Force on Practice Guidelines. Circulation 2007; 116: e418-99.

6. Struthers R, Erasmus P, Holmes K, Warman P, Collingwood A, Sneyd JR. Assessing fitness for surgery: a comparison of questionnaire, incremental shuttle walk, and cardiopulmonary exercise testing in general surgical patients. Br J Anaesthesia 2008; 101(6): 774-80.

7. Solway S, Brooks D, Lacasse Y, Thomas S. A Qualitative Systemic Overview of the Measurement Propertios of Functional Walk Tests Used in the Cardiorespiratory Domain. Chest 2001; 119:256-270.

8. Butland RJ, Pang J, Gross ER, et al. Two-, six, and 12-minute walking tests in respiratory disease. BMJ 1982; 284:1607-1608.

9. Bassey EJ, Fentem PH, MacDonald IC, et al. 
Self-paced walking as a method for exercise testing in elderly and young men. Clin Sci Mol Med 1976; 51:609-612.

10. Singh SJ, Morgan MDL, Scott S, et al. Development of a shuttle walking test of disability in patients with chronic airways obstruction. Thorax 1992; 47:1019-1024.

11. Revill SM, Morgan MDL, Singh SJ, et al. The endurance shuttle walk: a new field test for the assessment of endurance capacity in chronic obstructive pulmonary disease. Thorax 1999; 54:213-222.

12. Singh SJ, Morgan MD, Hardman AE, Rowe C, Bradley PA. Comparison of oxygen uptake during a conventional treadmill test and the shuttle walking test in chronic airflow limitation. Eur J Respir J 1994; 7: 2016-20.

13. Onorati P, Antonucci R, Valli G, et al. Noninvasive evaluation of gas exchange during a shuttle walking test vs. a 6-min walking test to assess exercise tolerance in COPD patients. Eur JAppl Physiol 2003: 89: 331-6.

14. Halm HA, Browner WS, Tubau JF, Tateo IM, Mangano DT. Echocardiography for assessing cardiac risk in patients having noncardiac surgery. Study of Perioperative Ischaemia Research Group. Ann Intern Med 1996; 125: 433-441.
15. Older P, Hall A. Clinical Review: How to identify high-risk surgical patients. Critical Care 2004; 8: 369-372.

16. Dunselman PH, Kuntze CE, van Bruggen A, Beekhuis H, Piers B, Scaf AH, Wesseling H, Lie K1: Value of New York Heart Association classification, radionuclide ventriculography, and cardiopulmonary exercise tests for selection of patients for congestive heart failure studies. Am Heart J 1988; 116: 1475-1482.

17. Pearse RM, Holt PJE, Grocott MPW. Managing perioperative risk in patients undergoing elective non-cardiac surgery. BMJ 2011; 343: d5759.

18. Older P, Hall A, Hader R. Cardiopulmonary exercise testing as a screening test for perioperative management of major surgery in the elderly. Chest 1999; 116:355-62.

19. Older P, Smith R, Courtney P, Hone R. Preoperative evaluation of cardiac failure and ischaemia in elderly patients by cardiopulmonary exercise testing. Chest 1993; 104: 701-4.

20. Gouvas N, Tan E, Windsor A, Xynos E, Tekkis P. Fast-track vs standard care in colorectal surgery: a meta-analysis update. Int J Colorectal Dis. 2009; 24: 1119-31 